\title{
Fully Automatic Kidneys Detection in 2D CT Images: A Statistical Approach
}

\author{
Wala Touhami, Djamal Boukerroui, and Jean-Pierre Cocquerez \\ HEUDIASYC, UMR CNRS \#6599, Université de Technologie de Compiègne, \\ BP 20529 - 60205 Compiègne Cedex, France \\ \{ouala.touhami, djamal.boukerroui, jean-pierre.cocquerez\}@hds.utc.fr
}

\begin{abstract}
In this paper, we focus on automatic kidneys detection in 2D abdominal computed tomography (CT) images. Identifying abdominal organs is one of the essential steps for visualization and for providing assistance in teaching, clinical training and diagnosis. It is also a key step in medical image retrieval application. However, due to gray levels similarities of adjacent organs, contrast media effect and relatively high variation of organ's positions and shapes, automatically identifying abdominal organs has always been a challenging task. In this paper, we present an original method, in a statistical framework, for fully automatic kidneys detection. It makes use of spatial and gray-levels prior models built using a set of training images. The method is tested on over 400 clinically acquired images and very promising results are obtained.
\end{abstract}

\section{Introduction}

Recently, a clinically operational Content-based image retrieval (CBIR) system, based on a semi-supervised learning approach and dedicated to help osteoarticular diagnosis on Magnetic Resonance (MR) images, is developed by our research team [1]. Needless to emphasis that the retrieval success is highly dependent on the images description stage. The purpose of our actual work is to develop an extension of the system to be applied to computed tomographic (CT) images of the abdomen, specifically to kidneys cysts. Unlike, the osteo-articular MR images, abdominal CT images present a higher variability. Indeed, different tissues (soft and hard) with different sizes and shapes across individuals and across slices may appear. This increases the complexity and the hardness of the image description step in a sense that a description index constructed using the whole image may be inefficient in the retrieval step. Salient features and relevant descriptors, in our application, are those which encode information about the kidney and the cysts. All other features, salient or not, could be considered as outliers. Therefore, the detection of the regions of interest (kidneys in our case) is a prerequisite step for the success of the retrieval process.

Automatically identifying organs from abdominal CT images series is challenging. Typically, the identification task is related to the segmentation problem, and few works treated this problem. For instance, Lee et al [2] solve the identification problem using fuzzy rules established based on knowledge of anatomy. 
Overlap information from consecutive slices is also used to guide the recognition process. Kobashi and Shapiro [3] integrate the recognition process with the segmentation one. The authors use anatomy knowledge to dynamically constrain a thresholding based segmentation. Here too shape constraints and overlap information from consecutive slices are used. These methods operate on 3D data and, to our knowledge, on healthy organs. The problem we address in this paper is a fully automatic detection of kidneys with cysts in a 2D CT slices. The presence of cysts makes the task more difficult because of the considerable increase of shape variability and intensity inhomogeneity. This discards the use of active shape models (ASM) or even appearance models (AAM) to guide the identification process. The construction of a probabilistic atlas is a potential alternative (see eg. [4]). Unfortunately, we are considering the problem in 2 dimensions 1 and the slice level may differ significatively making the approach unsuitable as different organs may appear/disppear. Besides this type of methods requires elastic registration, which adds a computational complexity to the problem.

In this paper, an original solution to the problem is proposed in a statistical framework. The outline of this paper is as follows. First, we start by the description of the mathematical modeling of the detection method and by specifying the different terms of the optimization problem to be solved. Section 3 describes the preprocessing steps and the models building. Section 4 presents results on clinically acquired data, and the summary and conclusion appear in Section 5.

\section{Modeling}

Our approach for the automatic kidneys detection makes use of spatial and gray-levels prior models built using a set of training images. Then, the detection problem is solved as a minimization of a cost function that takes into account the observed image and the prior models. This is described in details next.

\subsection{Prior Information}

Let $\Omega$ be the image spatial support and $\mathcal{L}$ denotes the set of image gray-level values. We suppose that each image is a realization of a random field $Y=$ $\left\{Y_{s}, s \in \Omega\right\}$ and we define a binary random field $X=\left\{X_{s}, s \in \Omega\right\}$ where $X_{s}=1$ means that the pixel $s$ is in the Kidney and $X_{s}=0$ otherwise.

- Spatial prior model: Given a set of $N$ training images $\left\{y^{i}, i=1 \ldots N\right\}$, we can construct on each image $i$, a realization $x^{i}$ of the random field $X^{i}$ by segmenting manually the kidney regions. Then, we empirically estimate a spatial prior pdf, $p_{K}(s)$, of the kidney areas using the mean field as follow:

$$
p_{K}(s)=\frac{\sum_{i=1}^{N} \frac{x_{s}^{i}}{n_{i}}}{N},
$$

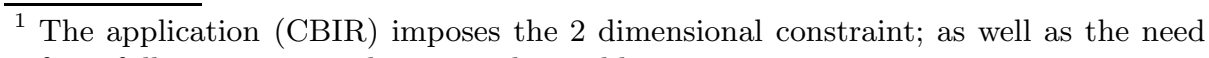
for a fully automatic solution to the problem. 
where $n_{i}$ is the number of pixels of the $i^{t h}$ image such as $x_{s}^{i}=1$. Using the above equation, we can build a spatial prior pdf for the left and right kidneys $p_{K_{l}}$ and $p_{K_{r}}$ respectively.

- Gray-level prior: We define $\omega$ a random variable taking values in $\mathcal{L}$ and we suppose that the random field $Y$ is an ergodic process in the same tissue (ie. $\left.X_{s}=1\right)$. In the same manner as the spatial model, we can build empirically a prior pdf of the kidney's gray-level values. Given the above assumptions, the prior gray-level pdf, $p_{\omega}(w)$ is position independent and is given by:

$$
p_{\omega}(w)=\frac{1}{N} \sum_{i=1}^{N}\left\{\frac{1}{n_{i}} \sum_{s / x_{s}^{i}=1} \delta\left(y_{s}^{i}-w\right)\right\} .
$$

Here $y_{s}^{i}$ is the gray-level value of pixel $s$ in image $i$ and $\delta$ is the Delta function.

\subsection{Cost Function Definition}

The proposed kidney identification method takes the form of an optimization of a cost function in order to detect two square windows $R_{l}$ and $R_{r}$, of side length $2 n+1$, respectively in the left and right kidneys. The general form of the cost function $J\left(R_{l}, R_{r}\right)$, to be minimized, is defined as follow:

$$
J\left(R_{l}, R_{r}\right)=\sum_{j=\{l, r\}}\left\{D_{\text {int }}\left(R_{j}\right)+D_{\text {spat }}\left(R_{j}\right)\right\}+S_{\text {sym }}\left(R_{l}, R_{r}\right) .
$$

Here, $D_{\text {int }}\left(R_{j}\right)$ is the data term for region $R_{j}, D_{\text {spat }}\left(R_{j}\right)$ is the spatial prior probability of region $R_{j}$ and $S_{s y m}\left(R_{l}, R_{r}\right)$ is a similarity term between the left and the right window. These terms will be detailed in the following paragraphs.

a) Data term: It measures how likely the gray-level distribution, $p_{\omega_{j}}(w)$, of the given region $R_{j}, j \in\{l, r\}$ is similar to the prior pdf $p_{\omega}(w)$ given by (eq. 2). This is a classical goodness-of-fit problem and several measures could be used. Two statistical measures are chosen, namely, Kuiper statistic $D_{K P}\left(p_{\omega}, p_{\omega_{j}}\right)$ and Kullback-Leiber divergence $D_{K L}\left(p_{\omega}, p_{\omega_{j}}\right)$.

b) Left/Right similarity measure: Recall that both square windows belong to the two kidneys and therefore are statistically dependant. Hence, we expect a high similarity between the two regions $R_{l}$ and $R_{r}$. Here too, several criterions could be used to measure this similarity. We select two measures, widely used by the computer vision community: the normalized cross-correlation $N C C\left(R_{r}, R_{l}\right)$ which measures the linear dependance and the mutual information $M I\left(R_{r}, R_{l}\right)$, which measures the statistical dependance of the two areas. Note that both measures have to be maximized 2 .

c) Spatial prior probability: Let $\Omega_{j}$ be the spatial support of the region $R_{j}$ and $S_{j}=\left\{s, p_{K_{j}}(s)>0\right\}, j \in\{r, l\}$. Using the spatial prior model (eq. 1), the probability that $\Omega_{j} \subset S_{j}$ is given by: $P\left(\Omega_{j}\right)=\sum_{s \in \Omega_{j}} p_{K_{j}}(s)$.

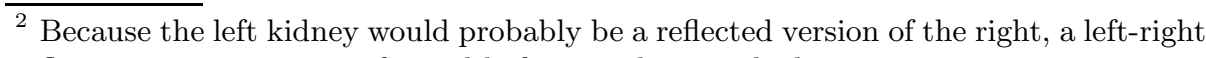
flipping operation is performed before similarity calculation. 
Now that we defined all the different terms, we rewrite eq. (3) as follow:

$$
J\left(R_{l}, R_{r}, \boldsymbol{\Theta}\right)=\sum_{j=\{l, r\}}\left\{D .\left(p_{\omega_{j}}, p_{\omega}\right)-\lambda_{1} \ln \left(P\left(\Omega_{j}\right)\right)\right\}-\lambda_{2} S .\left(R_{l}, R_{r}\right),
$$

where $D_{\text {. }}()=D_{K P}()$ or $D_{K L}(), S .()=N C C()$ or $M I()$ and $\lambda_{1} \in \mathbb{R}^{+}, \lambda_{2} \in \mathbb{R}^{+}$ are hyperparameters. The above cost function is minimized with respect to the variable $\boldsymbol{\Theta}=\left\{s_{l}, s_{r}\right\}$ representing the respective left and right window centroid.

The optimization algorithm employed uses the Nelder-Mead simplex search algorithm for multidimensional unconstrained minimization 5. It is deterministic method that attempts to minimize a scalar-valued function without any gradient information. The major problem of such local minimizer is the initialization. For our case, we choose to initialize the algorithm with 8 random candidates points with nonzero spatial pdf.

\section{Kidneys Detection Algorithm}

A block diagram of our new fully automatic kidneys detection approach is shown in Fig. 1. In this section we give algorithmic details about the preprocessing steps, the model building and the whole kidney delineation algorithm.

\subsection{Images Database}

All our images are routine acquisition in a public hospital. The images are acquired by several radiologists over 1 year time period (\# patients: 35 male, 12 female; age range: 19 to 88 years). In some cases a contrast media is administered, and 1 to 3 acquisitions are performed at different time delays. Consequently, there are images with injection of contrast media (+IV) and other without $(-\mathrm{IV})$. The images are acquired using different spatial resolutions (0.5703 to $0.9746 \mathrm{~mm}$ ) and slice thicknesses. An experienced radiologist selected 502 images (435 test images and 67 training images), representatives of the different cysts, which form our data set.

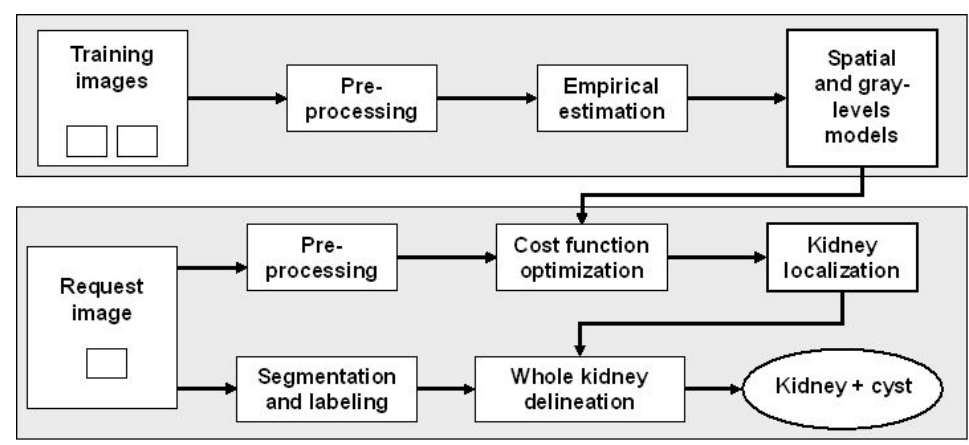

Fig. 1. Block diagram of the proposed automatic kidney detection method 


\subsection{Preprocessing}

In order to build or to make use of the spatial prior model, all the images must be in the same spatial referential. Ideally, all the images have to be elastically registered to a chosen reference image. Unfortunately, 2D elastic registration is not appropriate and will fail in several cases because of the high variability of the images content. Here, the variability is not only due to the soft nature of the tissues but also because of the significative variation, across individuals, of the position of the slice of interest. We found out that an accurate detection of the body area (without the skin and the fatty layer) is sufficient for our application. The detection algorithm and the resizing operation are detailed hereafter:

a) Region of interest (ROI) delineation:

A first threshold is used to detect the field of view and creates a binary mask, the exterior mask. Morphological operations (holes closing, opening and erosion) are then used to erase the staff table and reduce the mask in order to erase the skin. A second threshold, higher than the first one is chosen, to eliminate the fatty layer under the skin, followed by morphological operation to remove detected isolated pixels in the fatty layer. Finally, the two binary masks are combined and used to delineate the region of interest (ROI). We have tested this algorithm on the whole date base and found it very efficient with $100 \%$ success.

b) Resizing operation: Recall that the images in the database have different spatial resolutions. Hence, first a resizing operation is used to make all the ROIs have the same pixel size $\left(1 \mathrm{~mm}^{2}\right)$. The result, is a set of ROIs with a high variability of dimensions $\left(\mathrm{x}^{\mathrm{i}}, \mathrm{y}^{\mathrm{i}}\right)$ and more importantly with different aspect ratios. In order to put all the images in the same reference, we have to resize all the ROIs at a given fixed dimension $(\mathrm{x}, \mathrm{y})$. This operation will introduce shape distortions (i.e differences in axis resolution). We found that $(\hat{x}, \hat{y})=$ $\left(\mathbb{E}\left[\mathrm{x}^{\mathrm{i}}\right], \mathbb{E}\left[\mathrm{y}^{\mathrm{i}}\right]\right)$ is close to the optimal minimizer of the expected normalized quadratic error of the resolution differences between the two axis:

$\mathbb{E}\left[\left(\frac{\frac{x}{x^{1}}-\frac{y}{y^{1}}}{\frac{x}{x^{1}}+\frac{y}{y^{1}}}\right)^{2}\right], \quad$ where $\left(\frac{x}{x^{i}}, \frac{y}{y^{i}}\right)$ represent pixel resolutions.

\subsection{Models Building}

For the construction of the spatial and gray-levels prior models, we use a training set of 67 images $(30(-\mathrm{IV})$ and $37(+\mathrm{IV}))$, randomly selected from the database. The images are manually segmented and preprocessed as detailed above. Then, two spatial models are built separately, for the right and left kidney. Two graylevels pdfs are also built for the images with and without contrast media.

\subsection{Kidneys Detection}

Starting from the detected window $R_{l}$ (or $R_{r}$ ) inside the kidney, we utilize the corresponding segmentation map of the image in order to detect the whole 
kidney. The segmentation is performed, in a Bayesian framework, using a maximum a posteriori criteria (MAP) where the data term is modeled using a finite gaussian mixture model and the prior term is modeled by means of a hidden markov random field (HMRF). Specifically, we use the HMRF-Expectationmaximization algorithm, proposed by Zhang et al. 6]. However, the minimization is performed using a coarse to fine strategy based on a combination of a multiresolution model (for the observed data) and a multiscale model (for the hidden field) [7. The segmentation is fully automatic.

Our kidney delineation algorithm operates on the connected components of the segmentation map obtained using Suzuki's et al. algorithm [8]. An erosion operation on the fatty cluster - easily detected given its HU (Hounsfield Units) densitometric range - is first applied before the labeling operation in order to disconnect the kidney from other close organs (liver, spleen). This segmentation imperfection happens when the usual fatty layer surrounding the kidneys is too thin because of the closeness of the two organs. Hence, the delineation algorithm takes as input data the set of labels included in the previously detected window $R_{j}, j \in\{l, r\}$. Then, based on prior knowledge of abdominal anatomy, we define a set of IF THEN rules ( $\simeq 7$ rules) operating on a set of region's properties (related to shape, position, densitometric range, relative position, predefined prior models) in order to delineate the whole kidney. Briefly, we search for the bounding box that surely includes the kidney and the cysts, defined as the union of all the regions included in $R_{j}$ (the regions included in the defined bounding box constitute our candidates set). An adjusting operation utilizing the predefined rules is then performed. The selected regions constitute the kidney+cyst area.

\section{Results}

The proposed method is tested on the abdominal CT database detailed in section 3.1 (435 images, i.e without the training images). Illustrative detection results are shown in figure 2. The original images are viewed between -135 and $215 \mathrm{HU}$. The detected windows $\left(R_{l}, R_{r}\right)$, the rectangle delineating the whole kidney and the rectangle delineating the ROI are highlighted. We can observe images without (first 2 columns) and with (next 2 columns) contrast media. These examples illustrate the robustness of our automatic kidneys detection approach and underline the high variability of image content, in which the kidneys have neither the same appearance nor the same spatial positions. The last column shows typical cases of failure, mainly due to small kidneys (the circles show the correct kidneys location).

The algorithm presented in this paper has three free parameters that needed to be optimized, namely, $n$ for the window size and $\left(\lambda_{1}, \lambda_{2}\right)$ the tradeoffs between the different energy terms of the cost function. Experiments are carried out for different combinations of the statistical measures presented earlier in section 2.2 in order to choose the best parameter setting. To this end, we use the success rate as the selection criterion: we consider a case to be successful if a kidney (left or right) is correctly detected. That means that we calculate the success rate according to the number of kidneys not according to the number 

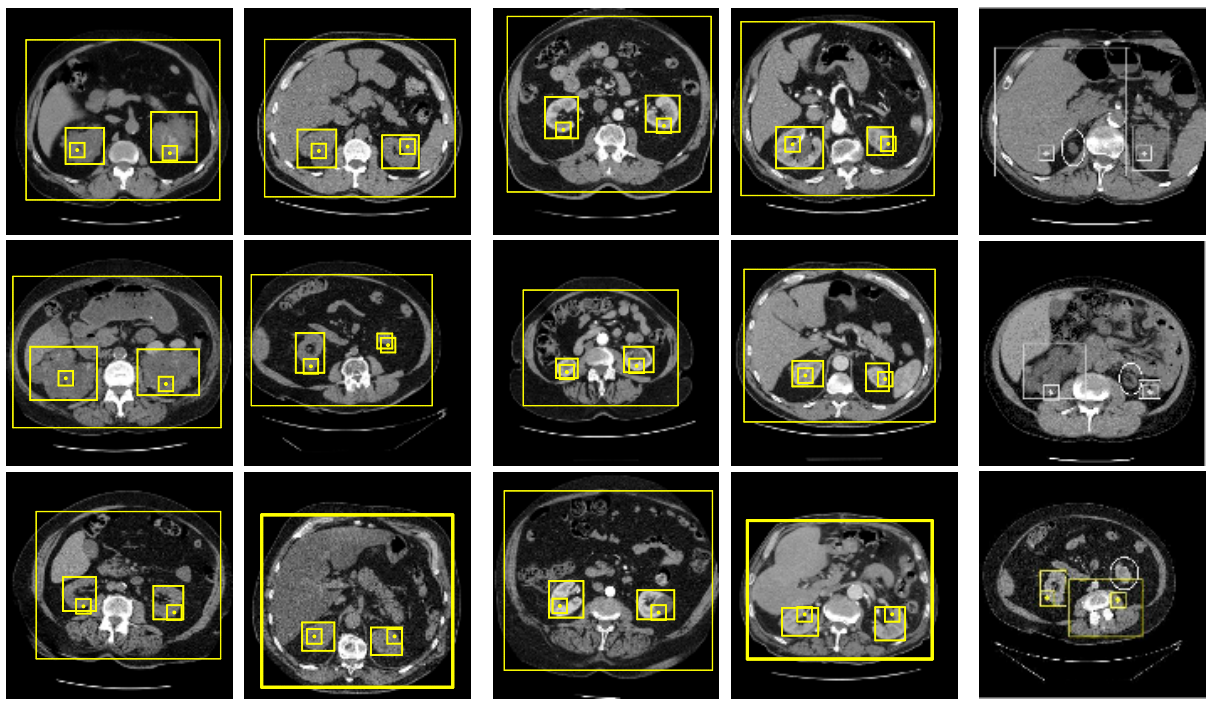

Fig. 2. Results of kidneys detection (see text for description)

Table 1. Best success rate of the window localization: comparison of different combinations of the statistical measures. $n=16$ is the best window size of all combinations.

\begin{tabular}{c|c|c|c|c} 
& \# images & & Kuiper test & Kullback test \\
\hline \hline \multirow{2}{*}{$(-\mathrm{IV})$} & \multirow{2}{*}{196} & NCC & $97.95 \%\left(\lambda_{1}, \lambda_{2}\right)=(1.0,0.00)$ & $97.44 \%\left(\lambda_{1}, \lambda_{2}\right)=(2.2,0.37)$ \\
\cline { 3 - 5 } & & MI & $97.44 \%\left(\lambda_{1}, \lambda_{2}\right)=(1.3,0.12)$ & $97.44 \%\left(\lambda_{1}, \lambda_{2}\right)=(2.2,0.37)$ \\
\hline$(+\mathrm{IV})$ & 239 & NCC & $95.78 \%\left(\lambda_{1}, \lambda_{2}\right)=(1.6,0.37)$ & $94.06 \%\left(\lambda_{1}, \lambda_{2}\right)=(2.2,0.00)$ \\
\cline { 3 - 5 } & & MI & $94.91 \%\left(\lambda_{1}, \lambda_{2}\right)=(1.4,0.37)$ & $94.72 \%\left(\lambda_{1}, \lambda_{2}\right)=(3.6,0.25)$ \\
\hline
\end{tabular}

of images. The three free parameters are optimized for each combination of the statistical measures (see table 1). Two conclusions can be drawn from this experiment. First, the success rates for the images (-IV) are significatively better than those of the images $(+\mathrm{IV})$ and this is true for all measures combinations of the statistical measures presented earlier. This result is expected considering the increase of heterogeneity caused by the injected contrast media. Second, there is no significant differences, in terms of success rate, between all the different combinations. However, we can argue that Kullback-Leiber divergence needs a higher weight for the spatial prior $\left(\lambda_{1}\right)$. Notice that the data term has always a weight of 1 in our formulation. We also present in Table 2 preliminary results, however promising, for the delineation of the whole kidney, which of course are expected to be lower than those for the window localization (about $-2 \%$; indicating an acceptable success rate for the delineation algorithm of about 95\%).

Finally, in order to asses the importance and the efficiency of the preprocessing step, we carried experiments in the same conditions as for the results shown in table1, however, without the preprocessing step. We recorded a drop of performances of about $20 \%$ for all combinations. 
Table 2. Success rate detection for the Kuiper-NCC combination

\begin{tabular}{l|c|c|c} 
& \# images & Rate of $R_{j}$ localization & Rate of kidney detection \\
\hline \hline Kidneys (-IV) & 196 & $97.49 \%$ & $95.01 \%$ \\
\hline Kidneys (+IV) & 239 & $95.78 \%$ & $93.75 \%$ \\
\hline
\end{tabular}

\section{Conclusion}

In this paper, a fully automatic approach for kidneys detection in 2D abdominal CT images is proposed. It involved a two steps approach. A localization step, which make use of a statistical spatial and gray-levels priors, followed by delineation step of the whole organ. An efficient and fast preprocessing step was also proposed in order to make all the images in the same spatial referential. The success rate of the proposed method was satisfactory; especially for images without contrast media (above 97\%). Therefore, this approach will be useful to compute relevant descriptors of the regions of interest (kidney + cysts) to be utilized in a CBIR system. Further work is in progress in order to improve the detection on images with contrast media (currently $\simeq 95 \%$ ). We mainly focus on improving the optimization method and on defining a confidence measure for the detection process by automatically identifing false detections. In addition, while in the current work, the main focus was on the localization of the kidney, in future work, we will concentrate on improving the kidney delineation algorithm.

Acknowledgements: We are grateful to Prof. A. Remond and to all the medical staff of the radiology department of CHU of Amiens for the data acquisition. This work was funded by 'Contrat de Plan Etat/Région Picardie 2000-2006'.

\section{References}

1. Najjar, M., Ambroise, C., Cocquerz, J.P.: Image retrieval using mixture models and EM alogorithm. In: 13th Scandinavian Conf. SPIE (2003) 1114-1121

2. Lee, C.C., Chung, P.C., Tsai, H.M.: Identifying multiple abdominal organs from CT image series using a multimodule contextual neural network and spatial fuzzy rules. IEEE Trans. Inf. Technol. Biomed. 7 (2003) 208-217

3. Kobashi, M., Shapiro, L.G.: Knowledge-based organ identification from CT images. Pattern Recognit. 28 (1995) 475-491

4. Park, H., Bland, P.H., Meyer, C.R.: Construction of an abdominal probabilistic atlas and its application in segmentation. IEEE TMI 22 (2003) 483-492

5. Press, W.H., Teukolsky, S.A., Vetterling, W.T., Flannery, B.P.: Numerical Recipes in C: The Art of Scientific Computing. Cambridge University Press, NY (1992)

6. Zhang, Y., Brady, M., Smith, S.: Segmentation of brain MR images through a hidden Markov random field model and the expectation-maximization algorithm. IEEE TMI 20 (2001) 45-57

7. Boukerroui, D., Baskurt, A., Noble, J.A., Basset, O.: Segmentation of ultrasound images - multiresolution 2D and 3D algorithm based on global and local statistics. Pattern Recognit. Lett. 24 (2003) 779-790

8. Suzuki, K., Horiba, I., Sugie, N.: Linear-time connected-component labeling based on sequential local operations. Comput. Vis. Image Underst. 89 (2003) 1-23 